\title{
Tuning ferroelectricity of polymer blends for flexible electrical energy storage applications
}

\author{
Xin Zhang ${ }^{1,2^{*}}$, Yanda Jiang ${ }^{1}$, Ruoqi Gao ${ }^{1}$, Xinhui $\mathrm{Li}^{1}$, Zhonghui Shen ${ }^{1}$, Bao-Wen $\mathrm{Li}^{1}$, \\ Qingfeng Zhang ${ }^{3}$, Shujun Zhang ${ }^{4}$ and Ce-Wen $\mathrm{Nan}^{5^{*}}$
}

\begin{abstract}
Ferroelectric polymers are the mainstay of advanced flexible electronic devices. How to tailor the ferroelectric polymer films for various applications via simple processing approaches is challenging. Here we demonstrate the tuning of ferroelectric responses can be achieved in polymer blends of poly(vinylidene fluoride-trifluoroethylene) (P(VDF-TrFE)) and polymethyl methacrylate (PMMA) prepared via a simple two-step process. The proposed two-step process endows the polymer blends with a random distribution of $\mathrm{P}(\mathrm{VDF}-\mathrm{TrFE})$ crystalline phase, hence decoupling the coherent ferroelectric domain interactions between continuous ordered crystalline phases that ubiquitously existed in common P(VDF-TrFE) film. The incorporation of the miscible non-crystalline PMMA chains with low-polarity results in reversal dipoles and a transition from ferroelectric to antiferroelectric-like behavior, overcoming the trade-off between the polarization and depolarization fields. In particular, resultant excellent mechanical and electrical properties of the polymer blend films give rise to remarkably improved breakdown strength and energy storage performance, surpassing $P(V D F-T r F E)$ and commercial biaxial-oriented polypropylene films. This work provides a simple and effective strategy to tailor the ferroelectric response of polymeric materials with great potential for flexible electrical energy storage applications.
\end{abstract}

Keywords: ferroelectric polymers, antiferroelectricity, energy density, breakdown strength, dielectric

\section{INTRODUCTION}

Ferroelectric polymers have attracted substantial academic and industrial interests due to their low mass density, flexibility and easy processability compared with their ferroelectric ceramic counterparts, which enable flexible electronic devices towards applications including energy storage, data storage, sensing, actuation and solidstate cooling [1-4]. Poly(vinylidene fluoride) (PVDF) and its copolymer poly(vinylidene fluoride-trifluoroethylene) (P(VDF-TrFE)) exhibit the best-known ferroelectricity of polymeric materials by the high dipole moment and high packing density of $\mathrm{C}-\mathrm{F}$ bonds in the molecule chains [5]. The $\mathrm{C}-\mathrm{F}$ groups are aligned perpendicular to the main chains in the polar crystalline phase with all-trans configuration, which can be switched upon the external field with most of the orientations maintained after removal of the electric field, resulting in strong hysteresis behaviors with both high maximum electrical displacement $\left(D_{\max }\right)$ and high remnant electrical displacement $\left(D_{\mathrm{r}}\right)$ [6]. The large $D_{\mathrm{r}}$ finds great potential for flexible piezoelectric applications; however, some other applications, such as electrical energy storage, electrostriction and electrocaloric refrigeration, require low hysteresis with minimum $D_{\mathrm{r}}$ to output high operating efficiency [7-11]. As an example, electrical energy storage is one of the most promising applications of PVDF-based polymers due to their highest dielectric responses among numerous polymeric materials [12]; however, the concomitant high $D_{\mathrm{r}}$ with exaggerated energy loss $\left(U_{\text {loss }}\right)$ and low energy efficiency $(\eta)$ restrict their applications, and meanwhile,

\footnotetext{
${ }^{1}$ State Key Laboratory of Advanced Technology for Materials Synthesis and Processing, Center of Smart Materials and Devices, Wuhan University of Technology, Wuhan 430070, China

${ }^{2}$ International School of Materials Science and Engineering, Wuhan University of Technology, Wuhan 430070, China

${ }^{3}$ Hubei Key Lab of Ferro \& Piezoelectric Materials and Devices, School of Materials Science \& Engineering, Hubei University, Wuhan 430062, China

${ }^{4}$ Institute for Superconducting and Electronic Materials, Australian Institute of Innovative Materials, University of Wollongong, North Wollongong, NSW 2500, Australia

${ }^{5}$ School of Materials Science and Engineering, State Key Lab of New Ceramics and Fine Processing, Tsinghua University, Beijing 100084, China

"Corresponding authors (emails: zhang-xin@whut.edu.cn (Zhang X); cwnan@tsinghua.edu.cn (Nan CW))
} 
most of the energy loss is transferred into heat resulting in early failures, i.e., thermal breakdown, by the excessive heat accumulation after numbers of charge-discharge cycles [13].

There have been successful attempts to tailor the ferroelectric properties of polymers to improve their functionalities and enable broader applications. One effective approach is to break the ferroelectric domains of polymers into nanopolar regions, by which the ferroelectric nature of polymer can be tuned and transferred into relaxor ferroelectric with greatly suppressed $D_{\mathrm{r}}[2,14]$. Such approach was reported by modifying $\mathrm{P}$ (VDF-TrFE) polymer chains with high-energy electron irradiation $[15,16]$ or adding a third stiffer monomer into polymer chains $[17,18]$, where the defects can be created to break the ferroelectric domains. Of particular interest is that the anti-ferroelectric-like behaviors with double hysteresis loops were observed in some $\mathrm{P}(\mathrm{VDF}-\mathrm{TrFE})$ grafted or blocked copolymer systems, since the graft or block segments engineer the switching behaviors of polar crystalline phase of polymers $[8,19-21]$. It is worth noting that, those previously tailored ferroelectric behaviors were achieved by modifying the structure of $\mathrm{P}(\mathrm{VDF}-\mathrm{TrFE})$ molecular chains with complex and high-cost processes. Thus an effective simple method to tune the ferroelectric behaviors of ferro-polymers is in great demand. Polymer blends by direct mixing of ferro-polymer and a second component are one of the possibilities in this aspect. However, the progress from this blend approach is far from satisfied, where the polymer blends by controlling the composition, crystallization behaviors and interfaces issues enable weakened ferroelectricity or enhanced relaxor feature but fail to tune the ferroelectric nature of ferro-polymers [22-27]. To this end, here we demonstrate the feasibility of tuning the ferroelectric response in polymer blend by a facile two-step process. The proposed two-step process avoids the exclusion effect existing in polymer blend films by the evaporation process of solvent from the polymer/diluent system, which generates the random distribution of $\mathrm{P}(\mathrm{VDF}-\mathrm{TrFE})$ crystalline phase and breaks the coherent coupling of ferroelectric domain between ordered crystalline phases in spherulites. In such premise, the incorporation of non-crystalline polymethyl methacrylate (PMMA) component with low polarity overcomes the trade-off between local polarization and depolarization fields in crystalline phases, resulting in reversal dipoles and transition to antiferroelectric-like behavior. Finally, the desired double hysteresis antiferroelectric-like behaviors with greatly suppressed remnant polarization are acquired, together with remarkably improved breakdown strength, and the polymer blend films show great potential for electrical energy storage applications (as shown in Fig. S1).

\section{EXPERIMENTAL SECTION}

\section{Preparation of polymer blend films}

The P(VDF-TrFE) (Arkema, with $30 \mathrm{wt} \%$ of TrFE) and PMMA (Aladdin) were dissolved into $\mathrm{N}, \mathrm{N}$-dimethylformamide (DMF) and stirred for $48 \mathrm{~h}$. The homogeneous mixture was then transferred into a syringe, and the electrospinning was carried out under an electric field of $1.5 \mathrm{kV} \mathrm{cm}^{-1}$. A roller was used to collect the electrospun composite fibers, the collected fibrous mats were peeled out from the roller and hot-pressed at $200^{\circ} \mathrm{C}$ for $10 \mathrm{~min}$ to obtain dense films. The condensed polymer films were further heated at $200^{\circ} \mathrm{C}$ for $5 \mathrm{~min}$ and then quenched in ice water. The quenched films were dried at $40^{\circ} \mathrm{C}$ for $5 \mathrm{~h}$ in vacuum for the complete evaporation of water. For comparison, the polymer blend films were also prepared by the commonly used solutioncasting method, in which the polymer blend solutions were cast into films on glass substrates with a handy and facile scraper. The as-cast films were dried in vacuum at $40^{\circ} \mathrm{C}$, and further heated at $200^{\circ} \mathrm{C}$ for $5 \mathrm{~min}$ and then quenched in ice water.

\section{Microstructure characterization}

The morphology of electrospun polymer nanofibers, surface and cross-sectional morphology of polymer films were characterized with scanning electron microscopy (SEM, JSM 7610F Plus). The crystal structures of the polymer films were analyzed with X-ray diffraction (XRD, Rigaku Smartlab).

\section{Measurements of dielectric and ferroelectric properties}

Copper electrodes (4 $\mathrm{mm}$ in diameter and $50 \mathrm{~nm}$ in thickness) were coated on both sides of the polymer films. Dielectric permittivity and dielectric loss were measured with a HP 4294A precision impedance analyzer (Agilent) at room temperature with a frequency range from $10^{2}$ to $10^{6} \mathrm{~Hz}$ at $1 \mathrm{~V}_{\mathrm{rms}}$. Electric displacement-electric field loops were measured at $10 \mathrm{~Hz}$ with a multiferroic ferroelectric test system (Premier II, Radiant Technologies, Inc.) at room temperature, and the direct current (DC) leakage current densities (in $\mathrm{A} \mathrm{cm}^{-2}$ ) were also analyzed by $\nabla D \cdot$ Area/ $\nabla t$ with this ferroelectric test system. Electric breakdown tests were performed with a Dielectric Withstand Voltage Test System (Beijing Electromechanical Research Institute Supervoltage Technique) at 
a ramping rate of $200 \mathrm{~V} \mathrm{~s}^{-1}$ with a limit current of $5 \mathrm{~mA}$. The charge and discharge behaviors were measured by resistor-capacitance circuit performed by a capacitor charge-discharge test system (PK-CPR1701, PolyK Technologies, USA) with a high-voltage MOSFET switch, where the sample was charged by an electric field of $200 \mathrm{MV} \mathrm{m}^{-1}$ through the MOSFET switch and the stored energy was discharged to a load resistor of $100 \mathrm{k} \Omega$.

\section{Phase-field simulation}

A phase-field model was employed to calculate the microscopic local electric field distribution in the polymers. In this simulation, the order parameters $a$ and $b$ were used to define the crystalline phase ( $a=1$ represents the crystalline phase and whereas not $a=0$ ) and amorphous phase, and hence the phase-dependent permittivity $\varepsilon(r)$ can be written as

$\varepsilon(r)=\varepsilon^{a} a(r)+\varepsilon^{b} b(r)$.

The electric displacement $D(r)$ in polymers can be obtained by

$D(r)=\varepsilon_{0} \varepsilon(r) E(r)$,

where $\varepsilon_{0}$ is the dielectric permittivity of the vacuum, and $E(r)$ is the local electric field distribution determined by
$E(r)=E_{\text {app }}(r)+E_{\text {pol }}(r)-E_{\text {depol }}(r)$,

where $E_{\text {app }}(r), E_{\text {pol }}(r)$, and $E_{\text {depol }}(r)$ represent the applied, polarization and depolarization electric fields, respectively. The local electric field $E(r)$ can be obtained by solving the electrostatic equilibrium equation:

$\nabla D(r)=0$

and then the $\Delta E$ can be calculated as

$E_{\mathrm{pol}}(r)-E_{\mathrm{depol}}(r)=E(r)-E_{\mathrm{app}}(r)$.

\section{RESULTS AND DISCUSSION}

A facile two-step process is proposed to prepare the $\mathrm{P}$ (VDF-TrFE)/PMMA blend films as given in Fig. 1a. Taking the $\mathrm{P}(\mathrm{VDF}-\mathrm{TrFE}) / \mathrm{PMMA}(80 / 20 \mathrm{vol} \%)$ blend as an example, the $\mathrm{P}(\mathrm{VDF}-\mathrm{TrFE})$ and PMMA powders with a certain proportion were mixed and dissolved to solutions with proper viscosity, then the solutions were transformed to nanofibers via electrospinning as a prestep; afterward, the fibrous mats were collected and then subjected to hot-pressing to form dense and flexible film. As shown in the SEM image (Fig. $1 a_{1}$ ), the as-obtained polymer blend nanofibers with a diameter of $500 \mathrm{~nm}$ exhibit favorable morphology with smooth surface and bead-free, and the resultant polymer blend film with a
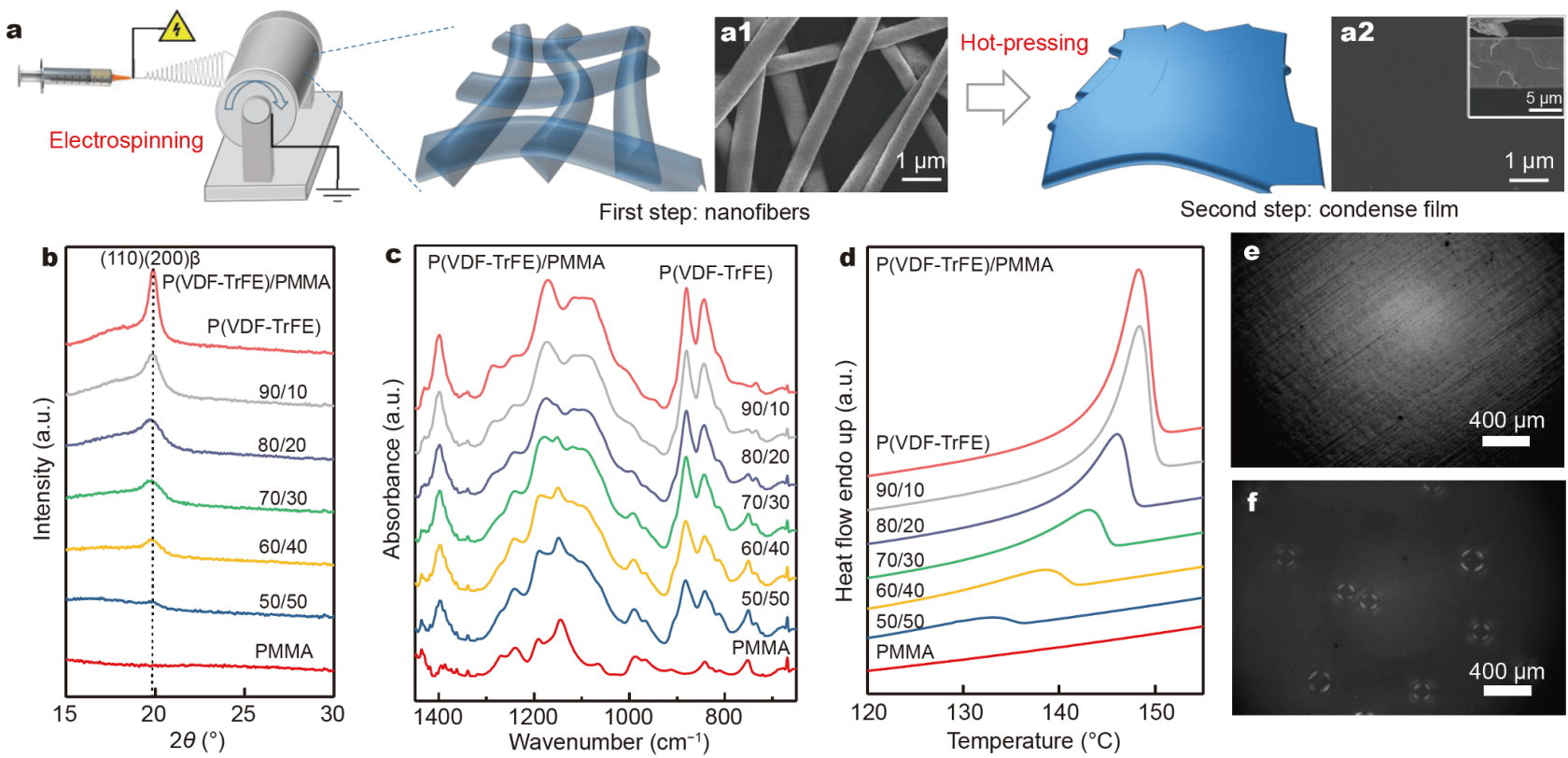

Figure 1 Ferro-polymer blend films prepared by the two-step process. (a) Schematic illustration of preparing $\mathrm{P}(\mathrm{VDF}-\mathrm{TrFE}) / \mathrm{PMMA}$ blend films via the facile two-step process: $\left(a_{1}\right)$ SEM image of as-electrospun polymer blend nanofibers and $\left(a_{2}\right)$ surface SEM image of the hot-pressed dense polymer blend film (inset is the cross-sectional SEM image of polymer film). (b) XRD patterns, (c) FTIR spectra and (d) differential scanning calorimetry (DSC) curves of pure polymers and polymer blends with different volume compositions. Optical microscopy images of polymer blend film prepared by (e) two-step process and (f) solution-casting mat. 
thickness of $10 \mu \mathrm{m}$ possesses high structural integrity without any voids or pores (Fig. $1 \mathrm{a}_{2}$ and Fig. S2). The crystalline structures of $\mathrm{P}(\mathrm{VDF}-\mathrm{TrFE})$ in both pure polymer film and blend film are mainly $\beta$ phase, as evidenced by the peaks at $2 \theta=19.7^{\circ}$ indicated by the XRD patterns of these polymer films (Fig. 1b), which is associated with $(110) /(200)_{\beta}$ reflection [27]. The dominant $\beta$ phase was also confirmed by the characteristic bands of all-trans configuration with absorbance peaks at 840 and $1280 \mathrm{~cm}^{-1}$ from the Fourier transform infrared spectroscopy (FTIR, Fig. 1c) [28]. In the $\beta$-phase P(VDF-TrFE), the $\mathrm{CF}_{2}$ groups are aligned in the same direction perpendicular to the polymer chains, leading to strong ferroelectricity with thermodynamic stable spontaneous polarization. With increasing contents of PMMA, however, the strength of the XRD peak gradually decreases and meanwhile the full width at half-maximum (FWHM) increases, demonstrating the reduced crystallinity and crystalline size, whereas the PMMA chains have not interpenetrated with the $\mathrm{P}(\mathrm{VDF}-\mathrm{TrFE})$ crystalline phase to change the intrinsic crystallographic structures. The melting temperature of the blend also consistently decreases with increasing amount of PMMA (Fig. 1d), indicating favorable miscibility between $\mathrm{P}(\mathrm{VDF}-\mathrm{TrFE})$ and PMMA molecular chains in the amorphous regions [29]. Of particular interest is that the growth of spherulites is confined and prevented by the formation of nanofiber pre-step in this unique two-step processing, where no visible spherulites can be observed in the blend film
(Fig. 1e), demonstrating the $\mathrm{P}(\mathrm{VDF}-\mathrm{TrFE})$ crystals are randomly distributed in the blends. On the contrary, the spherulites of $\mathrm{P}(\mathrm{VDF}-\mathrm{TrFE})$ were formed by ordered lamella crystals in the commonly used solution-casting approach directly from the evaporation of solvent, as indicated by the optical microscopy image (Fig. 1f).

According to these observations, a schematic illustration of the local microscopic polymer chain structures for two-step-processed $\mathrm{P}(\mathrm{VDF}-\mathrm{TrFE})$ and polymer blend are given in Fig. 2a. The $\mathrm{P}(\mathrm{VDF}-\mathrm{TrFE})$ is a semicrystalline polymer with coexistence of crystalline and amorphous phases, while in the polymer blend with addition of PMMA, the amorphous phase is mixed non-crystalline $\mathrm{P}(\mathrm{VDF}-\mathrm{TrFE})$ with PMMA molecular chains. Meanwhile, the two-step process generates a random distribution of crystalline phase which is encapsulated by amorphous phase. The unique structure of the polymer blend via the two-step approach is clearly distinguished from the common solution-casting-derived ones with spherulites (Fig. 2b), where the exclusion effect exists during the growth of spherulites from the polymer/diluent system; as a result, the non-crystalline PMMA tends to be excluded and mainly distributed away from the center of spherulites, while the ordered $\mathrm{P}(\mathrm{VDF}-\mathrm{TrFE})$ lamella crystals exist at the center area of spherulites [30-32].

In the polymer blends, the incorporated PMMA chains only interpenetrate with the amorphous region. Therefore, the local dielectric properties of the amorphous phase in the polymer blends are changed, as indicated by
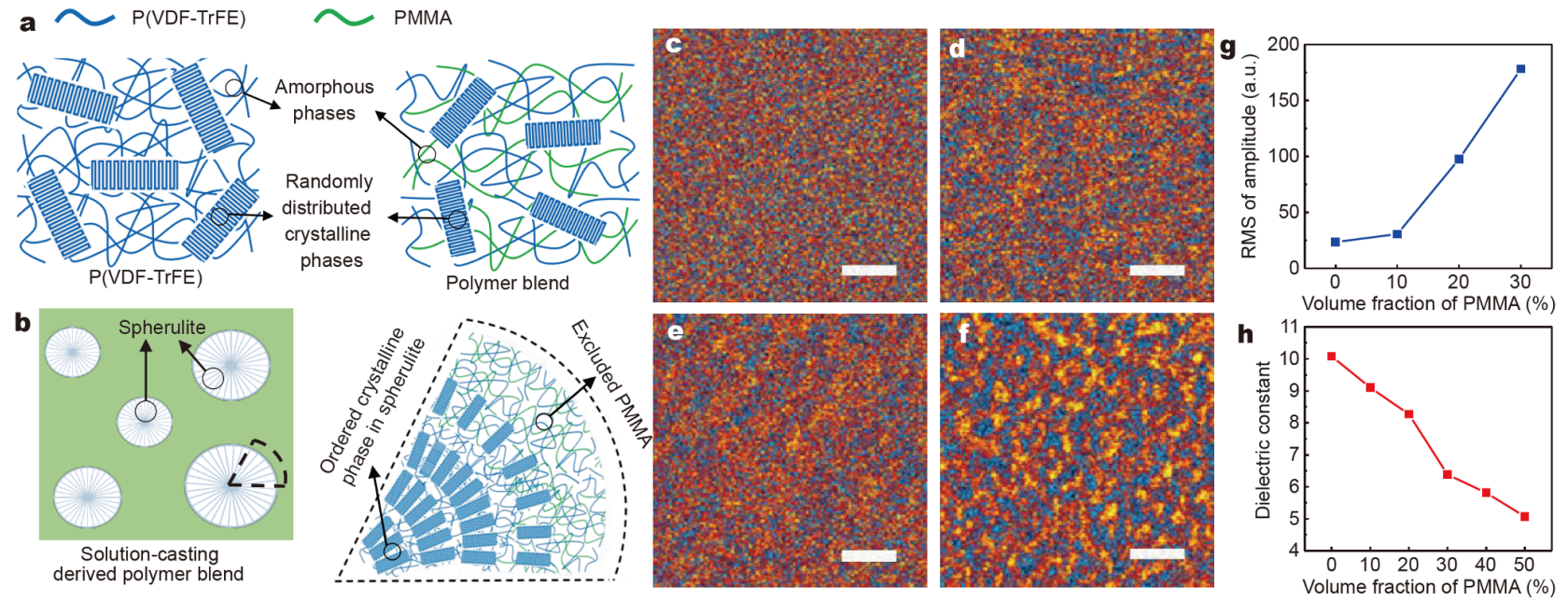

Figure 2 Microscopic polymer chain structures and local dielectric responses of polymer blends. (a) Schematic illustration of the polymer chain structures for two-step-processed $\mathrm{P}(\mathrm{VDF}-\mathrm{TrFE})$ and polymer blend. (b) Schematic illustration of the polymer chain structures for solution-castingderived polymer blend. PFM sequential electrical displacement responses of (c) pure P(VDF-TrFE) and polymer blends with (d) 10 vol\%, (e) 20 vol\%, (f) 30 vol\% of PMMA (scale bar, $200 \mathrm{~nm}$ for all). (g) The calculated RMS value of the mapped PFM electrical displacement amplitudes for polymer blends with different PMMA contents. (h) The variations of dielectric constant with the volume fraction of PMMA in the polymer blends. 
the mapped electrical displacement response of the polymer films via sequential excitation piezoresponse force microscopy (SE-PFM), as shown in Fig. 2c-f. Of particular significance is that the distribution of electrical displacements in polymer blends becomes more heterogeneous with stark contrast to the pure $\mathrm{P}(\mathrm{VDF}-\mathrm{TrFE})$. This trend can be further confirmed by the intense fluctuation of extracted line profiles (Fig. S3), and the increased root-mean-square (RMS) value of amplitude (Fig. 2g) which consistently increases from 23 to 98 for $\mathrm{P}(\mathrm{VDF}-\mathrm{TrFE})$ and the blend with 20 vol\% PMMA, respectively. The electrical displacement of the polymer under an external electric field is determined by two factors: the first is the local amount and orientation of dipoles; the other is the freedom and mobility of the polymer chains. In the pure $\mathrm{P}(\mathrm{VDF}-\mathrm{TrFE})$, the heterogeneity of electrical displacement mainly comes from the different polymer structures in amorphous and crystalline regions. After the incorporation of PMMA chains into the polymer blends, the contrast of electrical displacement between amorphous and crystalline regions is enlarged by the suppressed local electrical displacement in the amorphous phase. Two origins are responsible for this suppression: the PMMA possesses lower intrinsic molecule polarizabilities $\left(\varepsilon_{\mathrm{r}} \sim 4\right)$ than the $\mathrm{P}(\mathrm{VDF}-\mathrm{TrFE})\left(\varepsilon_{\mathrm{r}} \sim\right.$ 10 ), while the stiffer PMMA chains with high modulus decrease the local molecule mobilities of P(VDF-TrFE) after being interpenetrated, both leading to the suppressed dielectric response and electrical displacement in the amorphous phase. The SE-PFM sequential electrical displacement responses of the solution-casting-processed blend films were also tested for comparison as shown in Fig. S4. The solution-cast blend films exhibit markedly different distribution morphologies of electric displacement compared with that of two-step-processed ones; both the high-displacement and low-displacement regions are continuously distributed in the solution-cast blends, indicating the existence of continuous ordered $\mathrm{P}(\mathrm{VDF}-\mathrm{TrFE})$ lamella crystals by the exclusion of PMMA. The local electrical displacement behaviors in the twostep-processed blend films are in good agreement with the measured macroscopic dielectric constants. With increasing content of PMMA, the dielectric constant consistently declines from 10 to 5 for pure $\mathrm{P}(\mathrm{VDF}-\mathrm{TrFE})$ and polymer blends with 50 vol\% PMMA, respectively (Fig. $2 \mathrm{~h}$ and Fig. S5).

The suppressed dielectric responses in amorphous phase may also have a direct impact on the ferroelectric properties of the polymers. The ferroelectric responses of polymer blends and pure $\mathrm{P}(\mathrm{VDF}-\mathrm{TrFE})$ were investigated by measuring the electrical displacement $(D)$-electric field $(E)$ curves with a bipolar triangular wave at the frequency of $10 \mathrm{~Hz}$, the current I-electric field $E$ curves were also measured to understand the dipole switch behaviors since the switch of dipoles is always concomitant with remarkably increased current. As indicated in Fig. $3 a$, pure $\mathrm{P}(\mathrm{VDF}-\mathrm{TrFE})$ exhibits the typical features of ferroelectric hysteresis with rectangular-shaped $D-E$ loops. Two current peaks, i.e., P1 \& P1', from the first and third quadrants of the $I-E$ loops are also identified, which are induced by the orientation of polar ferroelectric domains of $\mathrm{P}(\mathrm{VDF}-\mathrm{TrFE})$ with increasing electric field reaching over the critical value, and then reversed with increasing negative electric field (Fig. 3e), corresponding to the coercive field. The incorporation of 10 vol\% PMMA in the polymer blend leads to slimmer $D-E$ loops (Fig. 3b) and much suppressed current peaks (Fig. 3f), indicating enhanced relaxor feature of $\mathrm{P}(\mathrm{VDF}-\mathrm{TrFE})$. However, the intrinsic ferroelectric nature remains since only two current peaks are identified from the $I-E$ curves. Remarkable changes appear with increasing content of PMMA to $20 \mathrm{vol} \%$, as shown in Fig. 3c, d, where the polymer blend shows notably distinguished double hysteresis $D-E$ loops with minimal $D_{\mathrm{r}}$, which is in stark contrast to the ferroelectricity but exhibits similar behavior as that of anti-ferroelectric. This can be confirmed by the four current peaks existing in the $I-E$ curves (Fig. 3g, h). Compared with the ferroelectric P(VDF-TrFE), the current peaks $\mathrm{P} 1$ and $\mathrm{P} 1$ ' are found shifting to higher electric field corresponding to the antiferroelectric to ferroelectric phase transition, while the additionally emerged two current peaks $\mathrm{P} 2$ and $\mathrm{P} 2$ ' from the second and fourth quadrant indicate the ferroelectric to antiferroelectric phase transition [33]. The suppressed $D_{\mathrm{r}}$ is further identified in Fig. S6. For pure P(VDF-TrFE), both the $D_{\max }$ and $D_{\mathrm{r}}$ consistently increase with increasing electric field. The $D_{\max }$ follows the same trend as the pure $\mathrm{P}(\mathrm{VDF}-\mathrm{TrFE})$, while the $D_{\mathrm{r}}$ shows minimal increase with increasing electric field up to $500 \mathrm{kV} \mathrm{mm}^{-1}$ for polymer blend with over $20 \mathrm{vol} \%$ of PMMA addition. It is interesting to note that the ferroelectric properties of the polymer blends are governed by polarization behaviors of amorphous phase with further increasing PMMA above $50 \mathrm{vol} \%$, where the polymer blends exhibit paraelectric properties with narrow hysteresis loops (Fig. S7). However, the tailored ferroelectric properties of polymer blends are further found greatly dependent on the typical two-step process; as seen from Fig. 3i, with the same $\mathrm{P}$ (VDF-TrFE)/PMMA composition of $80 \mathrm{vol} \% / 20 \mathrm{vol} \%$, the $D-E$ loop of the polymer blend film prepared by the 

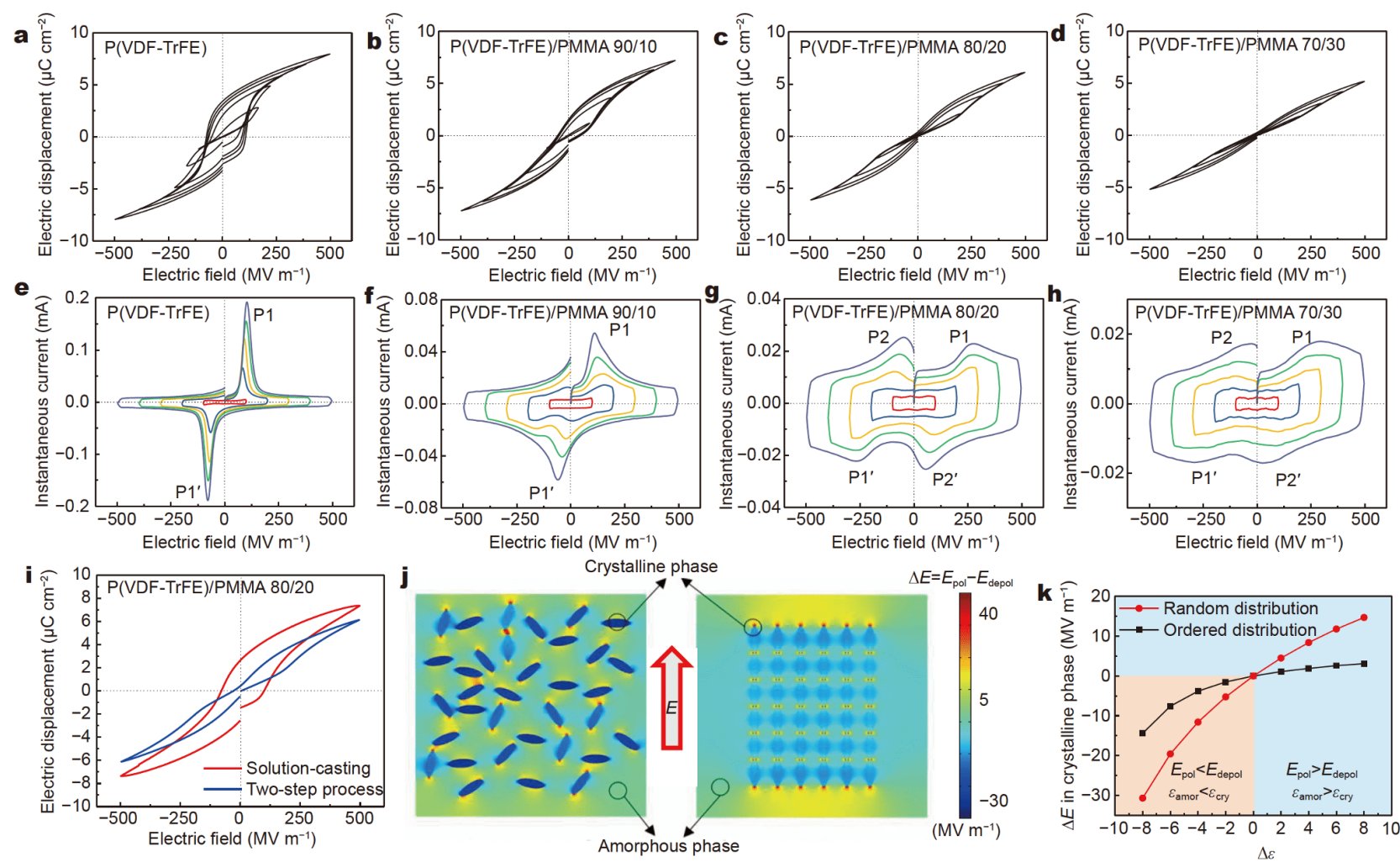

Figure 3 Ferroelectric properties of polymer blends. Bipolar electrical displacement-electric field $(D-E)$ loops of (a) pure P(VDF-TrFE) and polymer blends with PMMA contents of (b) 10 vol\%, (c) 20 vol\%, and (d) 30 vol\%. Bipolar current-electric field (I-E) loops of (e) pure P(VDF-TrFE) and polymer blends with PMMA contents of (f) $10 \mathrm{vol} \%$, (g) $20 \mathrm{vol} \%$, and (h) $30 \mathrm{vol} \%$. (i) Comparison of the $D-E$ loops of $80 \mathrm{vol} \% \mathrm{P}(\mathrm{VDF}-\mathrm{TrFE}) / 20 \mathrm{vol} \%$ PMMA blend prepared by solution-casting method and the two-step process. (j) Simulated microscopic local distribution of $\Delta E=E_{\mathrm{pol}}-E_{\mathrm{depol}}$ in polymer blend with random distribution and ordered distribution at the $E_{\text {app }}$ of $50 \mathrm{MV} \mathrm{m}^{-1}$. (k) Variations of calculated $\Delta E$ in crystalline phase with $\Delta \varepsilon=\varepsilon_{\text {cry }}-\varepsilon_{\text {amor }}$ in polymer blend with random distribution and ordered distribution.

solution-casting method with an evaporation process of solvent from the polymer/diluent system still exhibits obvious ferroelectric response.

It should be noted that the ferroelectric properties of the polymers are determined by polarization behaviors in both the crystalline and amorphous phases. As shown by the schematic model of polarization mechanisms in Fig. S8, the orientation of dipoles in crystalline phase produces a local polarization $\left(P_{\text {in }}\right)$, which is compensated by the polarization from the amorphous phase $\left(P_{\text {comp }}\right)$. Under the applied external electric field $\left(E_{\text {app }}\right)$, charge separation at the electrodes and the compensational polarization generate a local polarization field $E_{\mathrm{pol}}=\left(\varepsilon_{0} E_{\text {app }}\right.$ $\left.+P_{\text {comp }}\right) / \varepsilon_{0}$, where $\varepsilon_{0}$ is the vacuum dielectric constant. Meanwhile, the polarization of $P_{\text {in }}$ generates a depolarization field $E_{\text {depol }}=P_{\text {in }} / \varepsilon_{0}$ in the opposite direction. The trade-off between $E_{\text {depol }}$ and $E_{\mathrm{pol}}$ after the removal of the external $E_{\text {app }}$ dominates the switching behaviors of the dipoles and thus the ferroelectric properties of polymers.
Specifically, when $E_{\text {pol }}>E_{\text {depol }}$, the aligned dipoles maintain their orientations to produce strong ferroelectric hysteresis; on the contrary, part of the aligned dipoles can be reversed to the opposite direction when $E_{\text {pol }}<E_{\text {depol }}$ $[8,19,21]$. In the polymer blends, the local dielectric constant and polarization $\left(P_{\text {comp }}\right)$ from the amorphous phase decrease by incorporation of PMMA chains, which therefore reduces the $E_{\text {pol }}$ in crystalline phase and loses its competition with $E_{\text {depol }}$, and some aligned dipoles are thus reversed in the opposite direction antiparallel to the unreversed ones, finally leading to the antiferroelectric-like behaviors with greatly suppressed remnant electric displacement. Such a tailoring process is found highly relying on the random distribution of crystalline phase by the two-step process. When many crystalline phases are polarized upon the $E_{\text {app }}$, the ferroelectric domain couplings are generated within and between crystalline phases along the direction of $E_{\text {app }}[34,35]$. As mentioned, the spherulites are formed in the P(VDF-TrFE)/PMMA blend 
derived from the commonly used solution-casting method, where the adjacent ordered crystalline phase produces strong ferroelectric domain coupling interaction along the external field direction, screening the $E_{\text {depol }}$. By contrast, the proposed two-step process effectively generates the random distribution of crystalline phases which are encapsulated by the amorphous phases, thus breaking the coherent coupling between neighboring crystalline phases.

To better understand this proposed underlying physical mechanism related to the screening of electric field by local polarizations, the phase-field simulation method was employed to calculate the local distribution of $\Delta E=$ $E_{\text {pol }}-E_{\text {depol }}$ in P(VDF-TrFE)/PMMA blend after removal of $E_{\text {app }}$, where a lower dielectric constant of amorphous $\left(\varepsilon_{\text {amor }}\right)$ phase than that of crystalline phase $\left(\varepsilon_{\text {cry }}\right)$ is set to signify the reduced polarization in amorphous phase by PMMA incorporation. It is noted that ferroelectric domains cannot be formed in the amorphous phase with disordered structures, and thus the $\Delta E$ in crystalline phase with ordered aligned dipoles is critical to the ferroelectric properties of polymer. A negative $\Delta E$ in crystalline phase indicates smaller $E_{\mathrm{pol}}$ than the $E_{\mathrm{depol}}$, and thus the aligned dipoles in the crystalline phase can be reversed. The antiferroelectric-like property of polymer is acquired when a proper $\Delta E$ driving a necessary number of reversed dipoles in the opposite direction antiparallel to those unreversed dipoles. Fig. $3 \mathrm{j}$ compares the local $\Delta E$ in polymer blend with random distribution and ordered distribution. As seen, although both random and ordered distributions exhibit negative $\Delta E$ in the crystalline phase, the $\Delta E$ in crystalline phase is much higher with ordered distribution modeling on the local structure of spherulite, indicating the $E_{\text {depol }}$ is screened and mitigated in the ordered crystalline phase compared with random distribution. For further demonstrating the correlation between the $\Delta E$ and local dielectric constant, the calculated $\Delta E$ in crystalline phase is extracted and plotted with varied $\Delta \varepsilon=$ $\varepsilon_{\text {amor }}-\varepsilon_{\text {cry. }}$ According to Fig. $3 \mathrm{k}, \Delta \varepsilon<0$, i.e., a lower $\varepsilon_{\text {amor }}$ than $\varepsilon_{\text {cry }}$, safely leads to the $\Delta E<0$ in crystalline phase for polymer blend with both random and ordered distributions, and a lower $\Delta \varepsilon$ consistently decreases the $\Delta E$, resulting in higher degree of reversed dipoles. Compared with ordered distribution, the random distribution is much more capable to reduce the $\Delta E$ in crystalline phase and output stronger motivation for the reversed dipoles at the range of $\Delta \varepsilon<0$. For instance, the $\Delta E$ at $\Delta \varepsilon=-6$ is notably reduced from -7.6 to $-19.6 \mathrm{MV} \mathrm{m}^{-1}$ for ordered and random distribution respectively, which is a reduction of around $200 \%$. Whereas $\Delta \varepsilon<0$ also leads to $\Delta E<0$ in the ordered distribution, the very mild advantage of $E_{\text {depol }}$ to $E_{\text {pol }}$ fails to drive essential reversed dipoles in the crystalline phase. Moreover, the exclusion effect exists during the formation of spherulite in the P(VDF-TrFE)/ PMMA blend derived from the commonly used solutioncasting method; as a result, PMMA is mainly distributed away from the center of spherulites [32]. Therefore, the local $\varepsilon_{\text {amor }}$ cannot be effectively reduced in the spherulite to generate essential $\Delta \varepsilon<0$, where the continuous ordered crystalline phase still presents strong ferroelectricity. The two-step process avoids the exclusion effect and generates the random distribution of crystalline phase, leading to desirable negative $\Delta E$ and thus tailored ferroelectric response. A further cyclic test indicates the antiferroelectric-like property of the two-step-processed polymer blend is quite stable, as indicated by Fig. S9, since the $D-E$ loop of the polymer blend remains the same after $10^{4}$ cycles.

The incorporation of PMMA polymer chains via the proposed two-step process also leads to unexpected dielectric breakdown behaviors in the polymer blends. The breakdown strength of the polymer blends was analyzed with a two-parameter Weibull distribution function: $P(E)$ $=1-\exp \left(-\left(E / E_{\mathrm{b}}\right)^{\beta}\right)$, where $P(E)$ is the cumulative probability of electric failure, $E$ is experimental breakdown strength, $E_{\mathrm{b}}$ is a scale parameter refers to the breakdown strength at the cumulative failure probability of $63.2 \%$ which is also regarded as the characteristic breakdown strength, and $\beta$ is the Weibull modulus associated with the linear regressive fit of the distribution [36]. The $E_{\mathrm{b}}$ values of pure $\mathrm{P}(\mathrm{VDF}-\mathrm{TrFE})$ and polymer blends are given in Fig. 4a. The breakdown strength of pure P(VDF$\mathrm{TrFE}$ ) is characterized as $492.4 \mathrm{MV} \mathrm{m}^{-1}$, and in particular, with the incorporation of PMMA, the $E_{\mathrm{b}}$ is notably increased to $725.7 \mathrm{MV} \mathrm{m}^{-1}$ for the $80 \mathrm{vol} \% \mathrm{P}$ (VDFTrFE) $/ 20$ vol\% PMMA blend. The enhanced $E_{\mathrm{b}}$ of this blend is ascribed to the mutual contribution from the improved mechanical property and leakage current. Young's modulus of pure $\mathrm{P}(\mathrm{VDF}-\mathrm{TrFE})$ and polymer blends were calculated from the nanoindentation loaddisplacement curves (Fig. S10). As shown in Fig. 4b, compared with pure $\mathrm{P}(\mathrm{VDF}-\mathrm{TrFE})$, Young's modulus of the polymer blends is continuously improved with increasing content of stiff PMMA polymer chains, where the values are found to be 0.8 and $2.0 \mathrm{GPa}$ for $\mathrm{P}$ (VDFTrFE) and the polymer blends with $20 \mathrm{vol} \%$ of PMMA, respectively. Higher modulus of elasticity usually generates stronger endurance of field-induced electrostatic forces during the electro-mechanical breakdown, which hence gives rise to higher breakdown strength $[37,38]$. 

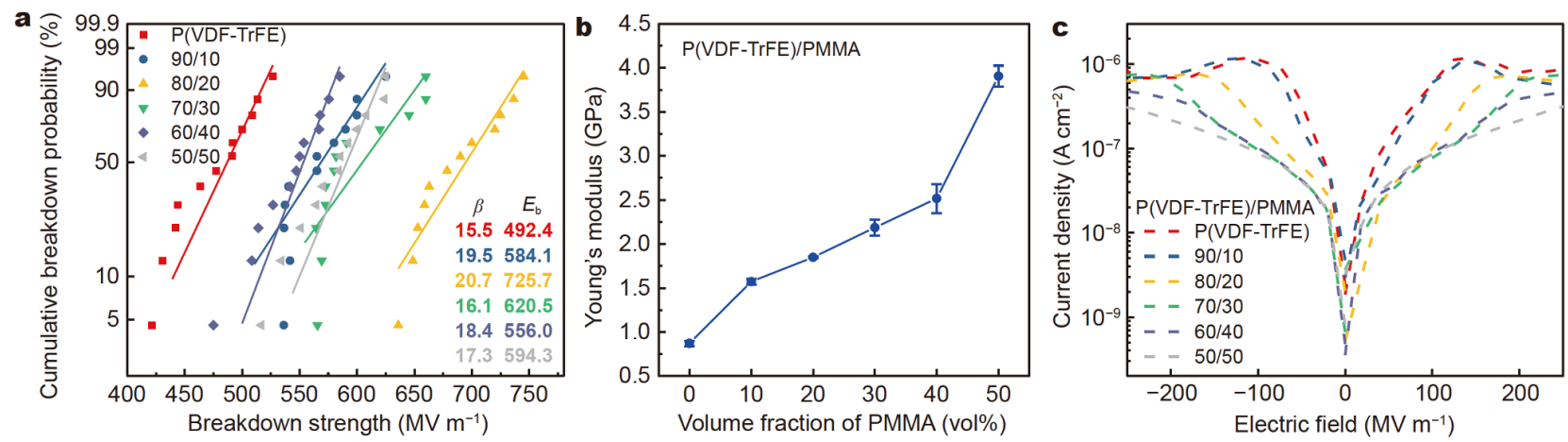

Figure 4 The breakdown strengths of polymer blends influenced by mechanical and electrical properties. (a) Failure probabilities of breakdown strength deduced from Weibull distribution for pure P(VDF-TrFE) and polymer blends. (b) Young's moduli for polymer blends with different contents of PMMA. (c) Variation of leakage current density with the electric field for pure P(VDF-TrFE) and polymer blends.

Another important factor impacting $E_{\mathrm{b}}$ of the polymer blends is the reduced leakage current density, where the current-induced Joule heating eventually leads to the thermal breakdown of the polymer [39]. As shown in Fig. $4 c$, the leakage current densities are much suppressed in the polymer blends compared with pure $\mathrm{P}(\mathrm{VDF}-\mathrm{TrFE})$. The reduced leakage current of blends may be ascribed to the modified interfaces between amorphous and crystalline phases, which can serve as traps for the mobile carriers. It is worth noting that with increasing content of PMMA, Young's modulus consistently increases and the leakage current consistently decreases; however, the $E_{\mathrm{b}}$ turns to decline with further increasing content of PMMA over $30 \mathrm{vol} \%$. The introduction of PMMA reduces the local dielectric constant in the amorphous phase and thus creates dielectric mismatch between amorphous and crystalline phases, which is enlarged by the high content of PMMA and leads to strong electric field accumulation at the amorphous/crystalline interfaces, being responsible for the declined $E_{\mathrm{b}}$ with the PMMA content above 30 vol\%.

Moreover, the two-step-processed polymer films generally exhibite higher Young's modulus, lower leakage current densities and higher breakdown strengths than the solution-cast polymer film counterparts for both pure $\mathrm{P}(\mathrm{VDF}-\mathrm{TrFE})$ and polymer blend (Fig. S11 and Table S1). For instance, Young's modulus is $1.67 \mathrm{GPa}$ for $80 \mathrm{vol} \%$ $\mathrm{P}(\mathrm{VDF}-\mathrm{TrFE}) / 20$ vol\% PMMA blend prepared by the solution-casting method, while a higher value of $1.85 \mathrm{GPa}$ is achieved in the two-step-processed one; the breakdown strength also increases from $607.5 \mathrm{MV} \mathrm{m}^{-1}$ for the solution-cast blend film to $725.7 \mathrm{MV} \mathrm{m}^{-1}$ for the two-stepprocessed blend film with the same $80 \mathrm{vol} \% / 20 \mathrm{vol} \%$ composition. The enhancement may be ascribed to the ultrahigh structural integrity and quality of hot-pressed condensed polymer films by the two-step process.

The double hysteresis ferroelectric behaviors and much-improved breakdown strength are expected to greatly benefit the electrical energy storage performances of the polymer blends. Therefore, the discharged energy density and $\eta$ of polymer films were calculated from the monopolar $D-E$ loops (Fig. S12) by the integration of dielectric displacement to the electric field and given in Fig. 5a and Fig. S13. As seen, all the polymer blends exhibit higher discharged energy density than the pure P(VDF-TrFE) (Fig. S13). The two-step-processed 80 vol\% $\mathrm{P}(\mathrm{VDF}-\mathrm{TrFE}) / 20$ vol\% PMMA blend possesses the highest discharged energy density of $20.4 \mathrm{~J} \mathrm{~cm}^{-3}$ (at an electric field of $720 \mathrm{MV} \mathrm{m}^{-1}$ ) among the studied polymers, which is an almost $40 \%$ enhancement over the solution-cast blend counterpart, and in particular $80 \%$ enhancement over pure $\mathrm{P}(\mathrm{VDF}-\mathrm{TrFE})$ films. Meanwhile, the two-stepprocessed polymer blend films are found to possess high $\eta$ above $70 \%$ (Fig. S13). Moreover, it is found that the two-step-processed polymer films generally possess higher discharged energy density and higher $\eta$ than the solution-cast counterparts of both pure $\mathrm{P}(\mathrm{VDF}-\mathrm{TrFE})$ and blend polymers (Fig. S11). For further demonstrating the reliability of the polymer blends serving as the dielectric energy storage medium, charge-discharge performances of the two-step-processed $80 \mathrm{vol} \% \mathrm{P}$ (VDFTrFE) $/ 20$ vol\% PMMA blend film were characterized. As given in Fig. 5b, both energy density and $\eta$ show no obvious degradation after charge-discharge cycling for up to $10^{6}$ cycles, indicating the high stability of the tailored ferroelectric behaviors and ultrahigh structural integrity of the polymer blend films. Furthermore, a fast chargedischarge test was performed to investigate the power density of the blend film. The polymer blend film with the 80 vol\% $\mathrm{P}(\mathrm{VDF}-\mathrm{TrFE}) / 20$ vol\% PMMA composition and 

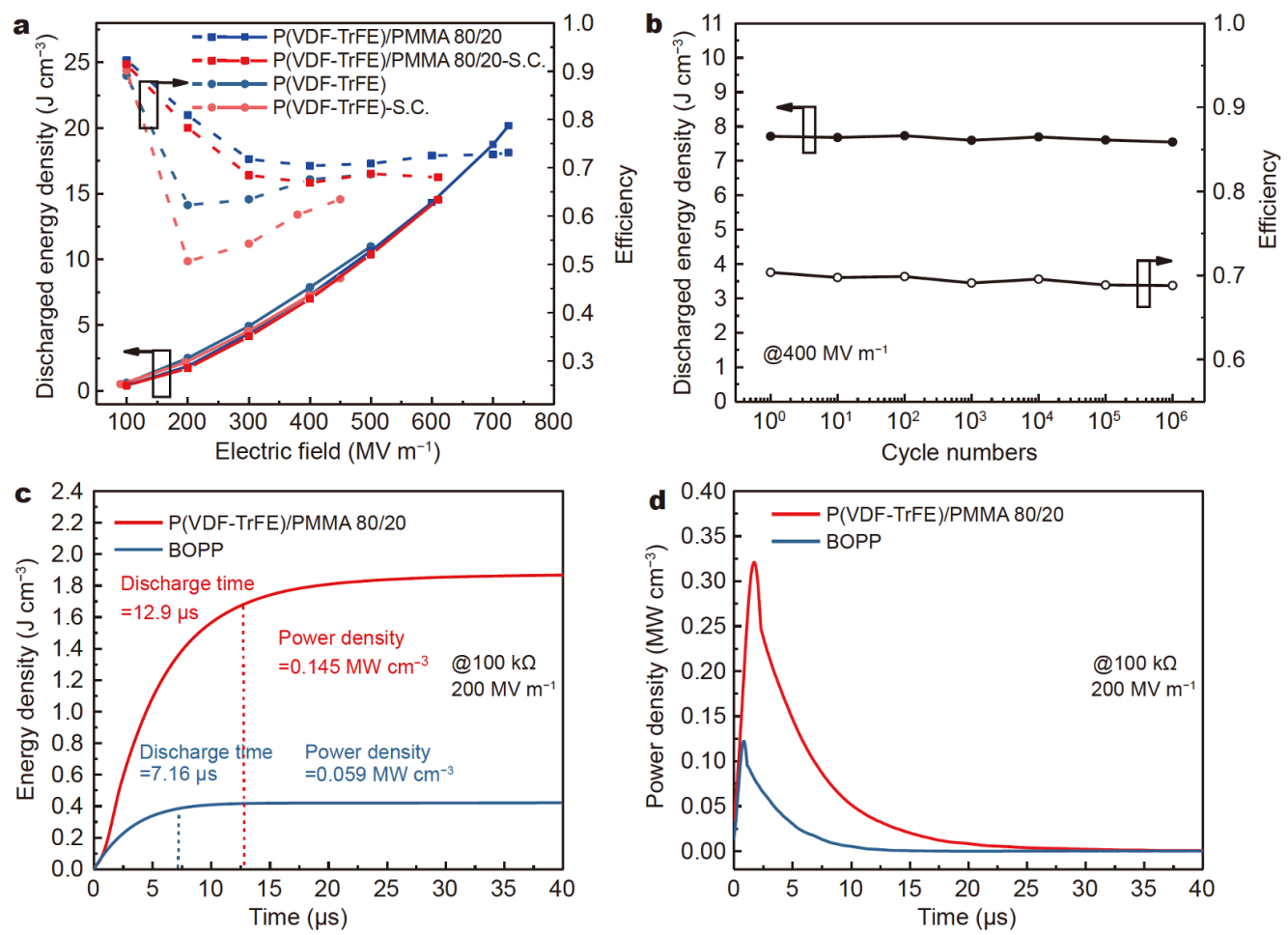

Figure 5 Energy storage properties of the polymer blends. (a) Variation of discharged energy density with electric field for pure P(VDF-TrFE), 80 vol\% P(VDF-TrFE)/20 vol\% PMMA blend films prepared by different methods. (b) Cyclic stability of energy density and energy efficiency for the two-step-processed 80 vol\% $\mathrm{P}(\mathrm{VDF}-\mathrm{TrFE}) / 20$ vol\% PMMA blend under $400 \mathrm{MV} \mathrm{m}^{-1}$. Variation of (c) discharged energy density and (d) power density on time of two-step-processed $80 \mathrm{vol} \% \mathrm{P}(\mathrm{VDF}-\mathrm{TrFE}) / 20 \mathrm{vol} \%$ PMMA blend and BOPP at the charged electric field of $200 \mathrm{MV} \mathrm{m}^{-1}$ and discharged to a load resistor of $100 \mathrm{k} \Omega$.

commercial biaxial oriented polypropylene (BOPP) film were charged by an electric field of $200 \mathrm{MV} \mathrm{m}^{-1}$ and then discharged to a load resistor of $100 \mathrm{k} \Omega$. The discharged time is determined as the time that $90 \%$ of the maximum energy density is discharged [40,41]. As shown in Fig. 5c, the commercial BOPP film releases an energy density of $0.4 \mathrm{~J} \mathrm{~cm}^{-3}$ within $7.16 \mu$ s, while in the blend film, an energy density of $1.7 \mathrm{~J} \mathrm{~cm}^{-3}$ is released within $16.2 \mu \mathrm{s}$. The determined highest power density of the blend film $\left(\sim 0.325 \mathrm{MW} \mathrm{cm}^{-3}\right)$ is around $260 \%$ that of commercial BOPP $\left(\sim 0.124 \mathrm{MW} \mathrm{cm}^{-3}\right)$ (Fig. 5d).

\section{CONCLUSIONS}

$\mathrm{P}(\mathrm{VDF}-\mathrm{TrFE}) / \mathrm{PMMA}$ ferro-polymer blend films with random distribution of crystalline phases have been prepared by a facile two-step process integrated with electrospinning and hot-pressing procedures. Compared with ferroelectric $\mathrm{P}(\mathrm{VDF}-\mathrm{TrFE})$, tailored ferroelectric behaviors with double hysteresis loops and muchsuppressed $D_{\mathrm{r}}$ have been achieved in the ferro-polymer blends. The underlying physical mechanism of the tailored ferroelectric properties is ascribed to the reduced $P_{\text {in }}$ of amorphous phases by incorporation of low-polarity PMMA chains, by which the polarization field in the crystalline phases is supressed to lose its competition with the depolarization field, resulting in reversal dipoles from crystalline phases. Meanwhile, the random distribution of crystalline phases is also confirmed as an essential factor impacting the ferroelectric properties of the polymer blends, which breaks the coherent coupling of ordered crystalline phase in spherulites. Moreover, the mechanical and electrical properties of the polymer blends are favorably improved in comparison to the pure $\mathrm{P}(\mathrm{VDF}-$ TrFE), which gives rise to much enhanced breakdown strength of the polymer blend films. The polymer blend film outputs an ultrahigh electrical energy storage density of $20.4 \mathrm{~J} \mathrm{~cm}^{-3}$ at $720 \mathrm{MV} \mathrm{m}^{-1}$, being $400 \%$ enhancement over the commercial BOPP film. This polymer blend approach by the two-step process provides a valid and effective strategy for tailoring the ferroelectric responses 
of polymeric materials, implying great potential for flexible electric energy storage applications.

\section{Received 20 August 2020; accepted 8 December 2020; published online 3 March 2021}

1 Liu Y, Aziguli H, Zhang B, et al. Ferroelectric polymers exhibiting behaviour reminiscent of a morphotropic phase boundary. Nature, 2018, 562: 96-100

2 Chu B, Zhou X, Ren K, et al. A dielectric polymer with high electric energy density and fast discharge speed. Science, 2006, 313: 334-336

3 Kim P, Doss NM, Tillotson JP, et al. High energy density nanocomposites based on surface-modified $\mathrm{BaTiO}_{3}$ and a ferroelectric polymer. ACS Nano, 2009, 3: 2581-2592

4 Prateek, Thakur VK, Gupta RK. Recent progress on ferroelectric polymer-based nanocomposites for high energy density capacitors: Synthesis, dielectric properties, and future aspects. Chem Rev, 2016, 116: 4260-4317

5 Lovinger AJ. Ferroelectric polymers. Science, 1983, 220: 1115-1121

6 Martins P, Lopes AC, Lanceros-Mendez S. Electroactive phases of poly(vinylidene fluoride): Determination, processing and applications. Prog Polym Sci, 2014, 39: 683-706

7 Meng N, Ren X, Santagiuliana G, et al. Ultrahigh $\beta$-phase content poly(vinylidene fluoride) with relaxor-like ferroelectricity for high energy density capacitors. Nat Commun, 2019, 10: 4535

8 Terzic I, Meereboer NL, Acuautla M, et al. Electroactive materials with tunable response based on block copolymer self-assembly. Nat Commun, 2019, 10: 1

9 Zhang G, Li Q, Gu H, et al. Ferroelectric polymer nanocomposites for room-temperature electrocaloric refrigeration. Adv Mater, 2015, 27: 1450-1454

10 Li Q, Zhang G, Zhang X, et al. Relaxor ferroelectric-based electrocaloric polymer nanocomposites with a broad operating temperature range and high cooling energy. Adv Mater, 2015, 27: 2236-2241

11 Zhang G, Zhang X, Yang T, et al. Colossal room-temperature electrocaloric effect in ferroelectric polymer nanocomposites using nanostructured barium strontium titanates. ACS Nano, 2015, 9: 7164-7174

12 Chen Q, Shen Y, Zhang S, et al. Polymer-based dielectrics with high energy storage density. Annu Rev Mater Res, 2015, 45: 433458

13 Li Q, Chen L, Gadinski MR, et al. Flexible high-temperature dielectric materials from polymer nanocomposites. Nature, 2015, 523: 576-579

14 Yang L, Li X, Allahyarov E, et al. Novel polymer ferroelectric behavior via crystal isomorphism and the nanoconfinement effect. Polymer, 2013, 54: 1709-1728

15 Cheng ZY, Zhang QM, Bateman FB. Dielectric relaxation behavior and its relation to microstructure in relaxor ferroelectric polymers: High-energy electron irradiated poly(vinylidene fluoridetrifluoroethylene) copolymers. J Appl Phys, 2002, 92: 6749-6755

16 Forsythe JS, Hill DJT. The radiation chemistry of fluoropolymers. Prog Polym Sci, 2000, 25: 101-136

17 Chu B, Xin Zhou, Neese, et al. Relaxor ferroelectric poly(vinylidene fluoride-trifluoroethylene-chlorofluoroethylene) terpolymer for high energy density storage capacitors. IEEE Trans Dielect Electr Insul, 2006, 13: 1162-1169
$18 \mathrm{Xu} \mathrm{H}$, Cheng $\mathrm{ZY}$, Olson $\mathrm{D}$, et al. Ferroelectric and electromechanical properties of poly(vinylidene-fluoridetrifluoroethylene-chlorotrifluoroethylene) terpolymer. Appl Phys Lett, 2001, 78: 2360-2362

19 Guan F, Wang J, Yang L, et al. Confinement-induced high-field antiferroelectric-like behavior in a poly(vinylidene fluoride-cotrifluoroethylene-co-chlorotrifluoroethylene)-graft-polystyrene graft copolymer. Macromolecules, 2011, 44: 2190-2199

20 Li J, Tan S, Ding S, et al. High-field antiferroelectric behaviour and minimized energy loss in poly(vinylidene-co-trifluoroethylene)graft-poly(ethyl methacrylate) for energy storage application. J Mater Chem, 2012, 22: 23468-23476

21 Guan F, Yang L, Wang J, et al. Confined ferroelectric properties in poly(vinylidene fluoride-co-chlorotrifluoroethylene)-graft-polystyrene graft copolymers for electric energy storage applications. Adv Funct Mater, 2011, 21: 3176-3188

22 Khan MA, Bhansali US, Almadhoun MN, et al. High-performance ferroelectric memory based on phase-separated films of polymer blends. Adv Funct Mater, 2013, 24: 1372-1381

23 Gao L, He J, Hu J, et al. Tailored ferroelectric responses and enhanced energy density in PVDF-based homopolymer/terpolymer blends. J Appl Polym Sci, 2014, 131: 40994

24 Chen XZ, Li X, Qian XS, et al. A polymer blend approach to tailor the ferroelectric responses in $\mathrm{P}(\mathrm{VDF}-\mathrm{TrFE})$ based copolymers. Polymer, 2013, 54: 2373-2381

25 Zhang X, Shen Y, Shen Z, et al. Achieving high energy density in PVDF-based polymer blends: Suppression of early polarization saturation and enhancement of breakdown strength. ACS Appl Mater Interfaces, 2016, 8: 27236-27242

26 Liu F, Li Z, Wang Q, et al. High breakdown strength and low loss binary polymer blends of poly(vinylidene fluoridetrifluoroethylene-chlorofluoroethylene) and poly(methyl methacrylate). Polym Adv Technol, 2018, 29: 1271-1277

27 Kim EJ, Kim KA, Yoon SM. Investigation of the ferroelectric switching behavior of P(VDF-TrFE)-PMMA blended films for synaptic device applications. J Phys D-Appl Phys, 2016, 49: 075105

28 Bae I, Kang SJ, Park YJ, et al. Organic ferroelectric field-effect transistor with $\mathrm{P}(\mathrm{VDF}-\mathrm{TrFE}) / \mathrm{PMMA}$ blend thin films for nonvolatile memory applications. Curr Appl Phys, 2015, 10: e54-e57

29 Aid S, Eddhahak A, Khelladi S, et al. On the miscibility of PVDF/ PMMA polymer blends: Thermodynamics, experimental and numerical investigations. Polym Testing, 2019, 73: 222-231

30 Tanaka H, Nishi T. New types of phase separation behavior during the crystallization process in polymer blends with phase diagram. Phys Rev Lett, 1985, 55: 1102-1105

31 Okada T, Saito H, Inoue T. Nonlinear crystal growth in the mixture of isotactic polypropylene and liquid paraffin. Macromolecules, 1990, 23: 3865-3868

32 Okabe Y, Murakami H, Osaka N, et al. Morphology development and exclusion of noncrystalline polymer during crystallization in PVDF/PMMA blends. Polymer, 2010, 51: 1494-1500

$33 \mathrm{Li} \mathrm{J,} \mathrm{Li} \mathrm{F,} \mathrm{Zhang} \mathrm{SJ.} \mathrm{Decoding} \mathrm{the} \mathrm{fingerprint} \mathrm{of} \mathrm{ferroelectric} \mathrm{loops:}$ Comprehension of the material properties and structures. J Am Ceram Soc, 2014, 97: 1-27

34 Zhu L, Wang Q. Novel ferroelectric polymers for high energy density and low loss dielectrics. Macromolecules, 2012, 45: 29372954

35 Stephanovich VA, Luk'yanchuk IA, Karkut MG. Domainenhanced interlayer coupling in ferroelectric/paraelectric superlattices. Phys Rev Lett, 2005, 94: 047601 
Zhang X, Shen Y, Zhang Q, et al. Ultrahigh energy density of polymer nanocomposites containing $\mathrm{BaTiO}_{3} @ \mathrm{TiO}_{2}$ nanofibers by atomic-scale interface engineering. Adv Mater, 2015, 27: 819-824

37 Li Q, Zhang G, Liu F, et al. Solution-processed ferroelectric terpolymer nanocomposites with high breakdown strength and energy density utilizing boron nitride nanosheets. Energy Environ Sci, 2015, 8: 922-931

38 Zhou X, Zhao X, Suo Z, et al. Electrical breakdown and ultrahigh electrical energy density in poly(vinylidene fluoride-hexafluoropropylene) copolymer. Appl Phys Lett, 2009, 94: 162901

39 Ieda; Ieda M. Dielectric breakdown process of polymers. IEEE Trans Elect Insul, 1980, EI-15: 206-224

40 Zhu Y, Zhu Y, Huang X, et al. High energy density polymer dielectrics interlayered by assembled boron nitride nanosheets. Adv Energy Mater, 2019, 9: 1901826

41 Jiang Y, Zhang X, Shen Z, et al. Ultrahigh breakdown strength and improved energy density of polymer nanocomposites with gradient distribution of ceramic nanoparticles. Adv Funct Mater, 2019, 30: 1906112

Acknowledgements This work was supported by the Basic Science Center Program of the National Natural Science Foundation of China (51788104), the National Natural Science Foundation of China (51802237, 52072280, 51872214 and 51872079), the Young Elite Scientists Sponsorship Program by CAST (2018QNRC001), the Open Fund of Hubei Key Laboratory of Ferro \& Piezoelectric Materials and Devices (K201807), and the Fundamental Research Funds for the Central Universities (193201002, 183101005 and 182401004).

Author contributions Zhang $\mathrm{X}$ and Nan CW conceived and supervised the study; Zhang X, Gao R and Jiang Y prepared the polymer films and performed the structural characterization; Jiang $\mathrm{Y}$ and Zhang $\mathrm{Q}$ carried out the measurements of energy storage properties; Li X and Zhang X conducted the PFM test; Shen $\mathrm{Z}$ performed the phase-field simulation; Zhang $\mathrm{S}$ and $\mathrm{Li}$ BW conducted the dielectric and ferroelectric measurements. Zhang X and Nan CW wrote the manuscript. All authors discussed the results and commented on the manuscript.

Conflict of interest The authors declare that they have no conflict of interest.

Supplementary information Experimental details and supporting data are available in the online version of the paper.

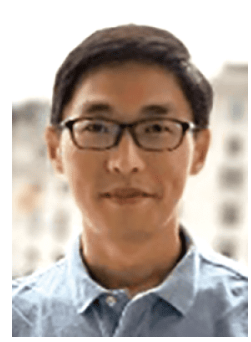

Xin Zhang joined the faculty of the International School of Materials Science and Engineering at Wuhan University of Technology, China, in July 2017. He received his BSc degree from Central South University, China, in 2012, and $\mathrm{PhD}$ degree in materials science and engineering from Tsinghua University in 2017. His research interests focus on the polymer nanocomposite materials for dielectric energy storage applications.

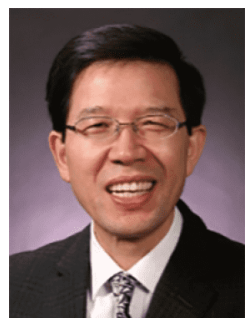

Ce-Wen Nan is a professor of the School of Materials Science and Engineering, Tsinghua University, Beijing, China. Before joining the faculty of Tsinghua University in 1999, he had worked at Wuhan University of Technology, China, since 1985. He was elected to the Chinese Academy of Sciences in 2011 and as a fellow of TWAS-the Academy of Sciences for the Developing World in 2012. His recent research focuses on polymer-based composites, multiferroic materials, solid state electrolytes, and thermoelectric oxide.

\section{聚合物共混薄膜的铁电特性调控及其柔性储能 应用}

张金金 ${ }^{1,2}$, 江彦达 ${ }^{1}$, 高若棋 ${ }^{1}$, 李昕蕙 ${ }^{1}$, 沈忠慧 ${ }^{1}$, 李宝文 ${ }^{1}$, 张清风 ${ }^{3}$, 张树君 ${ }^{4}$, 南策文 ${ }^{*}$

摘要 铁电聚合物在先进柔性电子器件中通常扮演重要角色, 如何 调控铁电聚合物薄膜的性能来满足其多样化应用是一个挑战. 本 文中，我们通过两步法制备了聚偏氟乙烯-三氟乙烯( P (VDF$\mathrm{TrFE})$ )与聚甲基丙烯酸甲酯(PMMA)的聚合物共混薄膜并对共混 薄膜的铁电特性进行了调控. 不同于常规 $\mathrm{P}(\mathrm{VDF}-\mathrm{TrFE})$ 薄膜中铁电 晶相的连续有序分布, 两步法工艺可以实现聚合物共混薄膜中 $\mathrm{P}(\mathrm{VDF}-\mathrm{TrFE})$ 晶相的随机分布, 进而打破常规铁电聚合物薄膜中相 邻晶相铁电畴间的耦合作用; 在此基础上, 共混薄膜非晶区内的低 极性PMMA组分可以通过降低去极化电场来调节偶极子的翻转行 为, 使共混薄膜由铁电特性转变为类反铁电特性; 此外, 铁电聚合物 共混薄膜优异的力学与电学性能可以显著提升其击穿强度, 最终 获得明显优于纯 $\mathrm{P}(\mathrm{VDF}-\mathrm{TrFE})$ 薄膜和商业双轴拉伸聚丙烯薄膜的 超高储能密度. 本论文提出了一种调控聚合物材料铁电特性的有 效策略, 为高储能密度铁电聚合物薄膜材料的发展带来巨大潜力. 